\title{
Comunicación
}

\section{Deficiencia de vitamina C como causa de mortalidady morbilidad en cuyes de crianza intensiva y su tratamiento}

\author{
Vitamin $\mathrm{C}$ deficiency as a cause of mortality and morbidity in intensive breeding \\ guinea pigs and their treatment
}

\section{Juan Olazábal L. ${ }^{\text {, Rosina Camargo H. }}$, Madeline García L. ${ }^{2}$ Siever Morales-Cauti ${ }^{3}$}

\section{Resumen}

Se describe la problemática de morbilidad y mortalidad de cuyes en una granja de la provincia de Trujillo, Perú. Por la historia clínica y las lesiones en piel se diagnosticó deficiencia de vitamina $\mathrm{C}$ y se realizó la suplementación de alfalfa ad libitum durante 21 días, colectándose muestras de sangre en cuatro animales los días $0,1,7,14$ y 21 de la suplementación. Se observó el incremento en los niveles de ácido ascórbico en sangre a partir del primer día de la suplementación y su normalización al final del periodo de evaluación, además de la completa recuperación de la condición corporal en los animales evaluados.

Palabras clave: cuyes; vitamina C; deficiencia; tratamiento

\section{AbSTRACT}

The problem of morbidity and mortality of guinea pigs in a farm in the province of Trujillo, La Libertad region, is described. Due to vitamin C levels, blood and lesions, a deficiency of vitamin $\mathrm{C}$ was diagnosed and therefore alfalfa ad libitum was supplemented during 21 days in four animals on days $0,1,7,14$ and 21 of the supplementations. The

${ }^{1}$ Laboratorio de Zootecnia y Producción Animal, Facultad de Medicina Veterinaria, Universidad Nacional Mayor de San Marcos, Lima, Perú

${ }^{2}$ Laboratorio de Patología Clínica, Facultad de Medicina Veterinaria, Universidad Nacional Mayor de San Marcos, Lima, Perú

${ }^{3}$ Laboratorio de Microbiología y Parasitología Veterinaria, Facultad de Medicina Veterinaria, Universidad Nacional Mayor de San Marcos, Lima, Perú

${ }^{4}$ E-mail: jolazaball@unmsm.edu.pe

Recibido: 19 de noviembre de 2018

Aceptado para publicación: 13 de junio de 2019 
increase in blood ascorbic acid levels was observed from the first day of the supplementation and its normalization at the end of the evaluation period. Moreover, the complete recovery of body condition in the evaluated animals.

Key words: guinea pig; vitamic C; deficiency, treatment

\section{INTRODUCCIÓN}

El ácido ascórbico (ascorbato, vitamina C) es uno de los más importantes antioxidantes solubles en agua que protegen a las células contra los efectos adversos del estrés oxidativo (Frei et al., 1989). Los animales domésticos tienen la habilidad de sintetizar vitamina $\mathrm{C}$ a partir de la glucosa por una serie de enzimas presentes en el hígado y riñón (Chatterjee, 1970). La enzima clave para que esta síntesis es la L-gulonalactona oxidasa que no está presente en humanos, cuyes y primates (Sato y Udenfriend, 1978).

La crianza del cuy (Cavia porcellus) ha suscitado un gran interés en el Perú, no solo por ser una valiosa fuente de nutrientes para el poblador rural, sino también como actividad económica que genera ingresos para el productor. Su relativa facilidad de crianza y demanda hace que esté en continuo incremento frente a otras especies pecuarias (De la Colina, 2017). La explotación de esta especie, especialmente en la costa peruana se ha intensificado y extendido, siendo actualmente una alternativa económica importante (Vidalón, 2014); sin embargo, los criadores de cuyes a este nivel afrontan el problema de reducción de áreas de producción de forraje verde, recurriendo a la adopción de la alimentación integral, buscando cubrir los requerimientos de los cuyes (Huamaní et al., 2016).

Este artículo tuvo como objetivo informar el caso de una granja de cuyes, donde se presentó un aumento de la morbilidad y mortalidad, habiéndose diagnosticado deficiencia de vitamina $\mathrm{C}$ y su corrección mediante la suplementación con al falfa(Medicago sativa).

\section{Descripción del Caso}

Un criador de cuyes de la provincia de Trujillo, Perú, se presentó al Laboratorio de Bioquímica, Nutrición y Alimentación Animal de la Facultad de Medicina Veterinaria (FMV) de la Universidad Nacional Mayor de San Marcos, Lima, manifestando que en su granja se venía presentando alta morbilidad y mortalidad de cuyes en diferentes etapas productivas y en ambos sexos.

El criador manifiesta que el problema se presenta desde hace ocho meses, aproximadamente. La población de cuyes era de 4200 y la alimentación se basaba en concentrado en polvo y acceso a agua de bebida. El concentrado era formulado y preparado en la granja mensualmente, para lo cual utilizaba afrecho de trigo, arrocillo, torta de soya y un suplemento comercial de vitaminas y minerales. Este alimento era complementado con chala fresca, de acuerdo con su disponibilidad.

Se evaluaron cuatro individuos (dos jóvenes, macho y hembra, y dos hembras adultas) que fueron trasladados a la FMV. Todos los animales se encontraban en pobre condición corporal. El productor indicó que la pérdida de peso era de manera paulatina. Los animales presentaban heridas en el rostro y miembros, alopecia, pelo hirsuto y pequeños abscesos en los miembros (Figura 1a, b, c). Los animales fueron pesados, se les tomó 1 $\mathrm{ml}$ de sangre de la vena cefálica, y fueron alojados en jaulas individuales, acondicionadas con comedero y bebedero. 

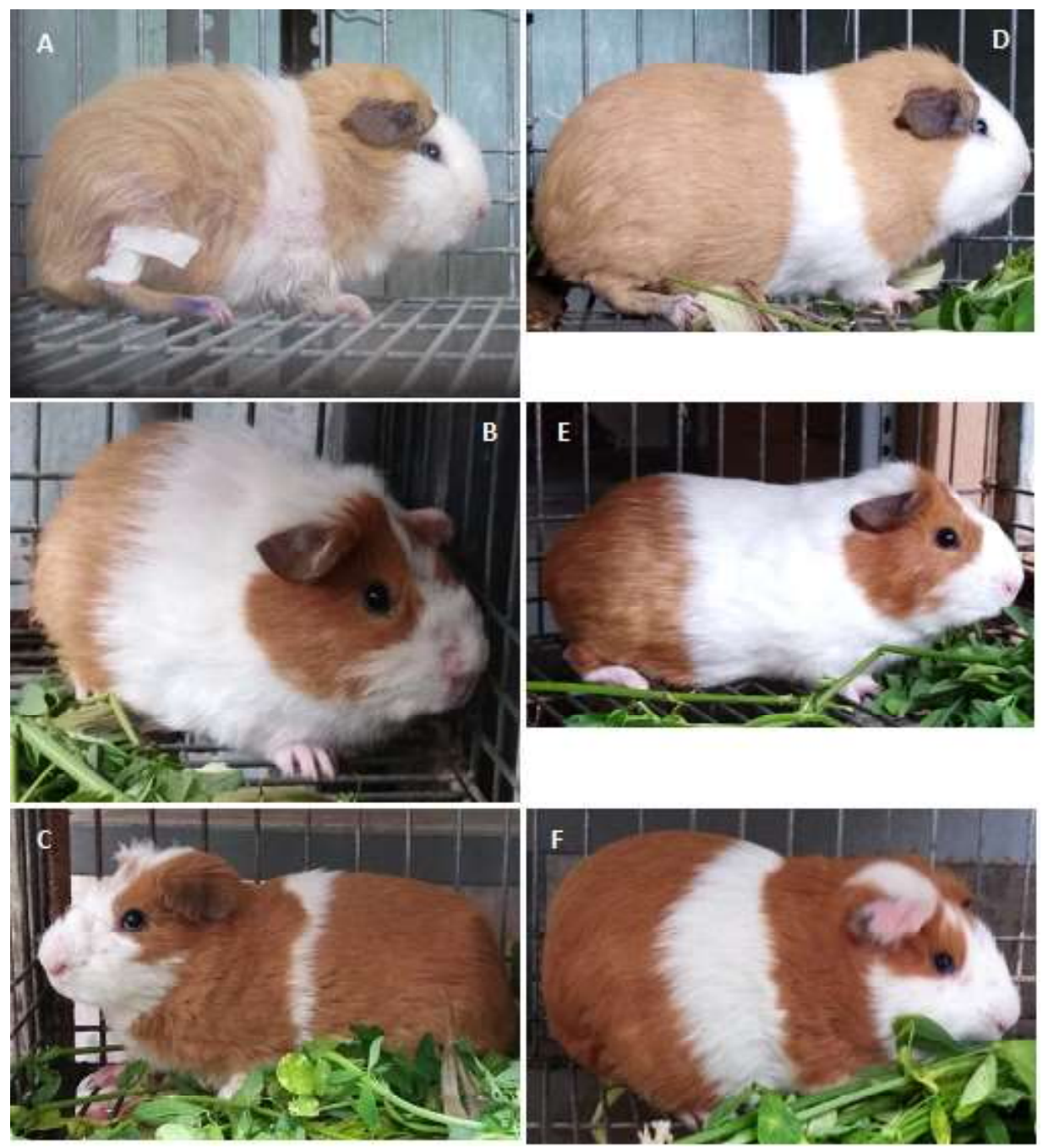

Figura 1. Cuyes afectados por deficiencia de vitamina $C$ de una granja de Trujillo, Perú, antes (A, B y C) y después (E, F, G) de la suplementación con alfalfa (Medicago sativa)

La muestra de sangre se usó para la determinación de ácido ascórbico, utilizando la metodología de Jagota y Dani (1982) inmediatamente a la toma de la muestra. La determinación se realizó por triplicado con un estándar de ácido ascórbico $(250 \mu \mathrm{g} / \mathrm{ml})$ y un blanco. Los resultados se expresaron como microgramos de ácido ascórbico por mililitro de suero $(\mu \mathrm{g} / \mathrm{ml})$.

Los cuatro individuos presentaron niveles muy bajos de ácido ascórbico (Cuadro 1), por lo que se procedió a alimentarlos con el concentrado que veían recibiendo en la gran- ja, pero suplementados con alfalfa fresca $a d$ libitum. El diagnóstico de la deficiencia de vitamina $\mathrm{C}$ se realizó a través de la historia clínica, signos y lesiones patológicas. Los niveles de ácido ascórbico en el suero sirvieron para confirmar el diagnóstico (Hawkins y Bishop, 2012). Se mantuvo el régimen de suplementación con alfalfa fresca durante 21 días, tomándose muestras de sangre y pesándose a los individuos los días $0,1,7,14$ y 21 .

En el Cuadro 1 se presenta la concentración de ácido ascórbico desde el inicio hasta el día 21 de evaluación. Se observa que, 
Cuadro 1. Niveles de ácido ascórbico $(\mu \mathrm{g} / \mathrm{ml})$ en cuyes afectados por deficiencia de vitamina $\mathrm{C}$ de una granja de Trujillo, Perú, desde el día 0 al día 21 de la suplementación con alfalfa (Medicago sativa)

\begin{tabular}{lccccc}
\hline \multirow{2}{*}{ Categoría } & \multicolumn{5}{c}{ Día } \\
\cline { 2 - 6 } & 0 & 1 & 7 & 10 & 21 \\
\hline Joven macho & 8.28 & 18.11 & 32.79 & 33.06 & 64.62 \\
Joven hembra & 9.41 & 15.66 & 23.92 & 27.55 & 45.11 \\
Hembra adulta 1 & 3.1 & 19.49 & 22.37 & 35.26 & 35.36 \\
Hembra adulta 2 & 3.1 & 20.61 & 27.0 & 29.31 & 76.81 \\
\hline Promedio & 5.97 & 18.47 & 26.52 & 31.29 & 55.47 \\
\hline
\end{tabular}

Cuadro 2. Peso vivo (g) de cuyes afectados por deficiencia de vitamina $\mathrm{C}$ de una granja de Trujillo, Perú, desde el día 0 al día 21 de la suplementación con alfalfa (Medicago sativa)

\begin{tabular}{lcccccc}
\hline \multirow{2}{*}{ Categoría } & \multicolumn{3}{c}{ Día } & \multicolumn{3}{c}{ Ganancia de peso } \\
\cline { 2 - 7 } & 0 & 7 & 14 & 21 & $\mathrm{~g} / \mathrm{d}$ & $\mathrm{g}$ \\
\hline Joven macho & 495 & 520 & 550 & 740 & 11.7 & 245 \\
Joven hembra & 560 & 596 & 640 & 770 & 10.0 & 210 \\
Hembra adulta 1 & 790 & 820 & 890 & 1085 & 14.0 & 295 \\
Hembra adulta 2 & 735 & 740 & 740 & 890 & 7.4 & 155 \\
\hline Promedio & 645 & 669 & 705 & 871 & 10.8 & 226 \\
\hline
\end{tabular}

previo a la suplementación, todos los individuos presentaron niveles inferiores de ácido ascórbico para la especie (Tveden-Nyborg et al., 2009), mientras que se incrementaron al segundo día de evaluación aproximándose al límite inferior del rango normal de la especie, y continuaron incrementándose durante el tiempo que duró la evaluación.

La evolución del peso vivo de los animales se presenta en el Cuadro 2. Se observa una ganancia total promedio de $226.3 \mathrm{~g}$ (rango: 155 - $295 \mathrm{~g}$ ) y una ganancia diaria promedio de $10.8 \mathrm{~g} / \mathrm{d}$ (rango: $7.4-14.0 \mathrm{~g} / \mathrm{d}$ ). La menor ganancia de peso se dio en la hembra adulta con mayores signos de desnutrición y la mayor ganancia de peso se registró en la segunda hembra adulta. Los cuatro in- dividuos se mostraron recuperados (Figuras 1d, e, f). La condición corporal mejoró notablemente en todos los individuos.

\section{Discusión}

La deficiencia de vitamina $\mathrm{C}$ es un problema común en la crianza de cuyes (Scott et al., 2001), pues no pueden sintetizarlo en su organismo y, por lo tanto, deben obtenerlo a través del alimento (Meredith, 2006). Los niveles subóptimos de vitamina $\mathrm{C}$ en humanos han sido asociados con un aumento de la morbilidad y mortalidad por enfermedades como el cáncer y enfermedades cardiovasculares (Frei et al., 2012). La deficiencia está asociada a lesiones de la piel, como petequias 
cutáneas, equimosis, hematomas, ulceraciones y seborrea generalizada (Scott et al., 2001) y están acompañadas de otros síntomas, como pérdida de peso, cojera, debilidad y gingivitis (Ellis y Mori, 2001).

Los signos de la deficiencia de vitamina $\mathrm{C}$ se pudieron observar en los cuatro individuos evaluados. Se encontró pelo hirsuto, baja condición corporal, heridas en los miembros y bajos niveles de vitamina $\mathrm{C}$ en sangre. La suplementación con alfalfa fresca permitió incrementar los niveles de vitamina C en sangre a partir del primer día de suplementación (Cuadro 1), recuperación de la condición corporal, cicatrización de heridas y brillo del pelaje. La vitamina $\mathrm{C}$ se puede administrar por vía parenteral a una dosis de 50 a $100 \mathrm{mg} /$ animal/día hasta que los signos desaparezcan (NRC, 1995).

La literatura menciona que la deficiencia de vitamina $C$ se puede presentar cuando la alimentación se realiza exclusivamente con una dieta comercial formulada para otra especie (Scott et al., 2001). Por otro lado, las dietas formuladas para cuyes son fortificadas con vitamina $\mathrm{C}$, pero el contenido se reduce por almacenamiento, luz, calor y humedad, pudiendo perderse hasta $50 \%$ de su actividad dentro de las seis semanas de almacenamiento (NRC, 1995), por lo que se recomienda utilizar el alimento dentro de los primeros días de su formulación y preparación (NRC, 1995).

La formulación de dietas para cuyes se hace usualmente tratando de cubrir todos los requerimientos nutricionales (NRC, 1995), información que ha sido generada en condiciones de laboratorio, y no en condiciones de crianza intensiva, donde se busca un máximo rendimiento productivo de los cuyes. Es por esto que es posible que los animales no lleguen a expresar su máximo potencial productivo debido a su relación con los niveles de vitamina $\mathrm{C}$.

\section{Literatura Citada}

1. Chatterjee IB. 1970. Biosynthesis of Lascorbate in animals. In: McCormick DB, Wright LD (eds). Methods in enzymology. San Diego, CA, USA: Academic Press. p 24-34.

2. De La Colina G. 2017. Evaluación del crecimiento compensatorio en cuyes de la raza Perú - Andahuaylas-2016. Tesis de Ingeniero Agrónomo. Abancay, Perú: Univ. Tecnológica de los Andes. $64 \mathrm{p}$.

3. Ellis C, Mori M. 2001. Skin diseases of rodents and small exotic mammals. Vet Clin N Am Exot Anim Pract 4: 493542. doi: 10.1016/S1094-9194(17)30041-5

4. Frei, B. Birlouez-Aragon I, Lykkesfeldt J. 2012. Authors' perspective: what is the optimum intake of vitamin $\mathrm{C}$ in humans? Crit Rev Food Sci 52: 815-829. doi: 10.1080/10408398.2011.649149

5. Frei B, England L, Ames BN.1989. Ascorbate is an outstanding antioxidant in human blood plasma. P Natl Acad Sci USA 86: 6377-6381. doi: 10.1073/ pnas.86.16.6377

6. Hawkins M, Bishop C. 2012. Disease problems of guinea pigs. In: Quesenberry KF, Carpenter JW (eds). Ferrets, rabbits, and rodents: clinical medicine and surgery. St. Louis, USA. p 295-310.

7. Huamani G, Zea O, Gutiérrez G, Vilchez C. 2016. Efecto de tres sistemas de alimentación sobre el comportamiento productivo y perfil de ácidos grasos de carcasa de cuyes (Cavia porcellus). Rev Inv Vet Perú 27: 486494. doi:10.15381/rivep.v27i3.12004.

8. Jagota SK, Dani HM. 1982. A new colorimetric technique for the estimation of vitamin $\mathrm{C}$ using folin phenol reagent. Anal Biochem 127: 178-182. doi: 10.1016/0003-2697(82)90162-2

9. Meredith A. 2006. Skin diseases and treatment of guinea pigs. In: Paterson $\mathrm{S}$ (ed). Skin diseases of exotic pets. Oxford: Blackwell Science. p 232-250. 
10. [NRC] National Research Council. 1995. Nutrient requirements of the guinea pig. In: Nutrient requirements of laboratory animals. $4^{\text {th }}$ revised ed. Washington DC, USA: National Academy Press. p 103-124.

11. Sato P, Udenfriend S. 1978. Scurvyprone animals including man, monkey, and guinea pig do not express the gene for gulonolactone oxidase. Arch Biochem Biophys 187: 158-162. doi: 10.1016/ 0003-9861(78)90018-8

12. Scott DW, Miller WH, Griffin CE. 2001. Dermatoses of pet rodents, rabbits, and ferrets. In: Muller and Kirk's small animal dermatology. $6^{\text {th }}$ ed.
Philadelphia: WB Saunders. p. 14151458.

13. Tveden-Nyborg P, Johansen LK, Raida Z, Villumsen CK, Larsen JO, Lykkesfelder J. 2009. Vitamin C deficiency in early postnatal life impairs spatial memory and reduces the number of hippocampal neurons in guinea pig. Am J Clin Nutr 90: 504-546. doi: 10.3945/ajcn.2009.27954

14. Vidalón J. 2014. Evaluación hematológica de dos líneas de selección de cuyes (cárnicos y precoces) criados en la Estación IVITA El Mantaro. Tesis de Médico Veterinario. Lima, Perú: Univ. Nacional Mayor de San Marcos. 98 p. 